\title{
DIE ANALYSE MORPHOLOGISCHE DES ADJEKTIVS IM ROMAN "EISPRINZESSIN"
}

\author{
Rutmaida \\ Rina Evianty \\ Jujur Siahaan
}

\begin{abstract}
AUSZUG
Das Ziel dieser Untersuchung ist es, um Morphologische die Adjektiven herauszufinden und die Adjektive im Roman 'Eisprinzessin' zu beschreiben. In dieser Untersuchung wird deskriptive qualitative Methode verwendet. Die Datenquelle dieser Untersuchung ist die Adjektive im Roman 'Eisprinzessin' von Carla Blumberg. Die Untersuchung verwendet die Tecknik der Datensammlung, um die zu kennen, zu markieren, zu versammeln, zu analysieren, und mit dem Phänomen angesammelten die Adjektive zu verallgemeinen. Die Ergebnisse dieser Untersuchung gibt es 253 die Morphologische Adjektive, nämlich 46 die Morphologische Adjektive mit dem Präfix, 167 die Morphologische Adjektive mit dem Suffix, 5 die Morphologische Adjektive mit dem Interfiks und 35 die Morphologische Adjektive mit dem Komposition.
\end{abstract}

Schlüsselwörter : Die Morphologische Des Adjektivs, Roman

\section{EINLEITUNG}

Im Deutschen gibt es einige Sprachelemente, nämlich; das Wort, der Satz, Formsatz, der Stuktursatz, Deklination, die Konjugation und andere.

Das Wort ist ein wichtiges Element bei der Sprachbildung, die eins in den Prozess der Herstellung der Wissenschaftlichearbeit. Chomsky (in Chaer 2011:163) stellt fest, dass das Wort die Grundlage ist, um ein Satz zu analysieren, nämlich Verben, Nomen und Adjektiv. Das Wort in einer Sprache wird einige Form gestallet, deshalb wird es die verschidene Bedeutung erzeugt. Morphologie ist die Linguistikwissenschaft, die über die Wortbildung oder Morphem erklärt.

In der Morphologie kann das Wort mit anderen Morphem vereinigen, um ein neues Wort zu bilden, das die Wortbedeutung verwandeln kann. Es gibt 3 Arten von Morphem, sie sind Prafix, Suffix und Infix.

Prafix liegt am Wortanfang und Suffix liegt am Ende des Wortes. Diese beide haben eine wichtige Rolle bei der Wortbildung. Ein Wort kann eine andere Bedeutung haben, nachdem ein Affixt eingeben wurde. Die Bedeutungveränderung 
wird den verschiedensten Wortklassen, nämlich das Nomen, das Adjektiv, das Verb und das Adverb geteilt. Aber diese Untersuchung beschränkt sich auf das Adjektiv. Das Adjektiv kann die Lage, der Nomen, Personen, Objeke und den Charakter einer Person beschreiben.

Beim Deutschlernen können über das Adjektiv gelernt wird. Im Deutschen können sich Adjektiv im Genus, Kasus, Numerus, Substantiv beziehen.

Das Adjektiv ist ein Wort, das eine genau Beschreibung von etwas auf Nomen in einem Satz erklärt bietet. Das Adjektiv ist eigene Wort von der Wortklasse und hat Morphologischenprozess. Der Morphologischenprozess des Adjektivs im Deutsch können geformt werden, wenn mit dem Affix zunehmen, nämlich un-, erz, -lich, -sam, -bar, -ig, -ös, -es-, -s-, -n-, -er-, -e-.

Man findet oft das Adjektiv im Roman, Gedicht, Lied, Märchen und die Unterhaltung im Alltag verwendet, um ein Dinge oder der Zustand erklärt. Aber diese Untersuchung beschränkt sich auf das Adjektiv im Roman.

Das Roman hat seit den 16. Jahrhundert zur beliebtesten epischen Großform in der Prosa entwickelt. (http://unidue.de/einladung/Vorlesungen/ epik/roman).

Der Roman im Leben ist sehr bekannt bei Jungen. Der Roman ist eine subjective Epik, in welcher der Verfasser sich die Erlaubnis ausbittet, die Welt nach seiner Weise darzustellen. Neben als die Lektüre und die Unterhaltung, der Roman kann als eine Untersuchung machen. Der Roman ist eine Abbildung das Lebens und ist ein Mittel um kulturelle Werte zu hinterlassen.

Im Welt gibt es vielen Roman bei Jungen, die Eltern und das Kind . Eines davon Roman ist der Roman Eisprinzessin. Die Jungen mögen der Roman Eisprinzessin, denn die Geschichte ist interessant, und kann Einblick hinzufügen. Die Untersucherin benutzt der Roman Eisprinzessin als Untersuchungobjekt, weil im Roman es die Werte Moralisierung gibt und viele Adjektiv zu untersuchen. Der Roman "Eisprinzessin" hat schon bereits Sternchen 5, 4, und 2 bekommt. Im Roman Eisprinzessin enthält Wertvorstellungen. Diese Werte sind Geduld, Glaube, 
Fleiß, Ehrlichkeit, Treue und Weisheit. Die Werte im Roman "Eisprinzessin" sind sehr wichtig für Studenten.

Beim Deutschlernen können Studenten Charakter gestaltet wird, wenn Lehrern/ Dozenten für einen guten Charakter-Bildung entsetzen. Deshalb können Lehrern/Dozenten einen Roman oder das Märchen als eines Medien für das Lernen Leseverstehen verwendet und die Moralischen im Roman als Form des Charakters diskutiert werden.

Basierend auf der obigen Beschreibung interessiert sich die Untersucherin dafür Adjektiv zu untersuchen. Der Titel dieser Untersuchung ist "Die Analyse morphologische des adjektivs im roman "Eisprinzessin von Carla Blumberg".

\section{THEORETISCHE GRUNDLAGE}

\section{Adjektiv}

Adjektive bezeichen Eigenschaften von Dingen oder Ereignissen, so zum Beispiel die Farbe (rot, blau), Form (rund, eckig), Gescmack (salzig), Temperatur (kalt) und Zugehörigkeit. Das Adjektiv ist ein Wort, das eine genaue Beschreibung von Nomen in einem Satz gibt. Elke Hentschel (2010:7) stellt fest, dass das Adjektiv ein Wort ist, um die Natur oder den Zustand des Menschen, Tiere und Dinge zu erklärt.

\section{Wortbildung}

Unter Wortbildungsaspekten kann man Adjektive in nicht von anderen Formen abgeleitete, wie arm, gro $\beta$ oder grün und solche unterteilen, die durch Worbildungsverfahren enstanden sind. Bei der Wortbildung von Adjektiven werden häufig Suffixe wie -bar, -haft, -ig, -isch, -lich, -sam usw. eingesetzt: einig, fruchbar, und andere. Aber auch Präfixe wie un oder miss (unschön, missvergnügt) kommen vor.

\section{Die Adjektivarten}


Engel (in Ending Khoerudin 2010:4) stellt fest, dass diese fünf Arten der Adjektive aufteilen. Es gibt: 1) Kuantifikatifadjektive, 2) Referensialadjektive, 3) Kualifikatifadjektive, 4) Klassifizierungadjektive, 5) Herkunftsadjektive

\section{Die Fuktion}

Elke Hentschel (2010:10) stellt dar, dass das Adjektiv drei Fuktion hat. Diese sind :

1) Attribute

Man können als Attribute verwendet werden und richten sich dann in Kasus, Genus, und Numerus nach dem Beziehungswort. Normelerweise stehen adjektive im Deutschen vor dem Substantiv.

\section{2) Prädikativ}

Das Fuktionverb oder Prädikativ fungiert als Prädikat entlang bestimmter Verben, wie sein, werden und bleiben. Prädikativ verwendete Adjektive, die bei einem Kopulaverb, zumeist sein stets unverändert.

\section{3) Adverbialbestimmung}

Schließlich können Adjektive auch als Adverbialbestimmung (auch; Adverbial) beim Verb auftreten. Sie bleiben dabei ebenfalls unverändert : Ma lachte laut.

\section{Morphologische Prozess}

Chaer Abdul (2012:177) stellt fest, dass Morphologische Prozess im Zusammenhang mit der Bildung von dem Wörtern durch den Prozess ein Zussamenführung ein Morphem mit andere Morphem betrefft. Es gibt veschiedene Morphologische Prozesse, in jeder Sprache, nämlich; Affix, Reduktion, Komposition, Suplesi, Modifikation, Zusammensetzung, Akronym, Clipping, und andere.

\section{a. Affix}

Elke Hentschel (2010:23) stellt fest, dass Affixe bedeutungstragende Elemente (Merpheme) sind, die nicht frei stehen können, sondern sich immer an 
ein anderes Elemente anheften müssen. Je nachdem, wo sie angefügt werden, heißen sie Präfix (Vorsilbe) und Suffix (Nachsilbe).

\section{a) Das Präfix}

Drosdowski (in Jujur Siahaan 2013:30) erklärt, dass das Präfix das vor ein Wort oder einen Wortstamm gesetztes unselbstständiges Wortbildungsmorphem eine Vorsilbe ist. Bei Adjektiven gibt es die Präfixe un und erz, aber in dem Roman 'Eisprinzessin' von Carla Blumberg gibt es nur -un. Das Präfix hat die Fuktion als "'negative", "schlecht'” und "schwer"auszudrichten.

\section{a) Das Suffix}

Das Suffix ist das Teil von dem Präfix, die enhalt am Endpotion in Grundform. Sufiks enthalt in Adjektiv sind; -lich, -sam, -bar, -ig, -ös.

\section{b) Interfiks}

Interfiks ist eine Art von Infix in den Prozess der Zusammenführung der beiden Elemente erscheint. Infix in der Adjektiv besteht aus; $-\boldsymbol{e s}$-, $-\boldsymbol{s}-,-\boldsymbol{n}$-,, $\boldsymbol{e r}$ - und $-\boldsymbol{e}$.

\section{b. Kompotion}

Fromkin (in Jujur Siahaan 2013:8) stellt dar, dass kompotion das Verbinden von zwei Grundformen ist, um ein neues Wort $\mathrm{zu}$ bilden. Neue Wörter möglichherweise Form durch andere Wörter zusammengesetze aneinanderreihen.

\section{Der Begriff der Roman}

Das Roman hat sich seit den 16. Jahrhundert zur beliebtesten epischen Großform in der Prosa entwickelt. (http://unidue.de/einladung/ Vorlesungen/ epik/roman). Goethe erklärt, dass der Roman uns mögliche Begebenheiten unter unmöglichen oder beinahe unmöglichen Bedingungen als wirklich darstellen soll. Der Roman ist eine subjective Epik, in welcher der Verfasser sich die Erlaubnis ausbittet, die Welt nach seiner Weise darzustellen. Der Roman ist ein literarische Werk, das in der Form der Narativ geschrieben ist und erzählen bestimmte Konflikte in der Charakter Gesichte aus dem Autorleben, weil in den Werken der 
Schrifsteller seine Geschichten, Gedanken, Gefühlen und Hoffnungen ausdrücken kann, (vgl. Nurgiyanto 2010:10).

\section{UNTERSUCHUNGSMETHODE}

In dieser Untersuchung wird die deskriptive qualitative Methode benutzt.

\section{Daten und Datenquelle}

Die Daten für die Untersuchung sind das Adjektiv in dem Roman Eisprinzessin von Carla Blumberg benutzen. Die Datenquelle dieser Untersuchung ist der Roman Eisprinzessin von Carla Blumberg.

\section{ERGEBNIS DER UNTERSUCHUNG}

\section{1) Das Affix}

Als Affixe gibt es im Roman 'Eisprinzessin' von Carla Blumberg das Präfixe, Suffixe, und Interfikse.

\section{a. Das Präfix}

In diesem Roman haben die Morphologischeprozess die Adjektiv mit dem Präfix -un, das Präfix -un + Suffix -lich, das Präfix - un + Suffix -ig und das Präfix -un + Suffix -bar. Das sind:
un+vermutet 'terduga'
unvermutet 'tak terduga
un+sympathisch 'simpati'
unsympathisch 'tidak simpati'
un+bemerkt 'mengetahui'
unbemerkt 'tanpa mengetahui'
un+deut(en)+lich 'jelas'
undeutlich 'tidak jelas, kabur'
un+verzüg(en)+lich 'langsung'
unverzüglich 'seketika'
un+heim+lich 'diam-diam'
unheimlich 'yang mengerikan'
$u n+r u h(e n)+i g$ 'tenang'
unruhig'ketidak nyamanan'
un+anständ+ig 'sopan-santun'
unanständig 'tidak sopan'
un+nachgeb+ig 'mudah mengalah'
unnachgiebig 'tidak mau mengalah'
$u n+f a s s(e)+b a r$ ' dapat dimengerti'
unfassbar 'tidak dapat dimengerti' 
un+(she)en +bar 'dapat kehilangan' unsichbar 'tidak kehilangan'

\section{b. Suffix}

Suffix im Roman ‘Eisprinzessin’ von Carla Blumberg gibt es -lich, -isch, -ig, bar, -sam, - ös, -los, und -voll.

\section{-lich}

Die Morphologische die Adjektive von Suffix -lich im Roman 'Eisprinzessin' von Carla Blumberg bestehen aus Nomen, Verben, Adjektiv, und Adverbia. Zum Beispiel:

$\begin{array}{ll}\text { der Freund 'teman'+lich } & \text { freundlich 'ramah tamah' } \\ \text { die Pein 'penderitaan' +lich } & \text { peinlich 'tidak enak' } \\ \text { ehr(en) 'menghormati' +lich } & \text { ehrlich 'jujur' } \\ \text { vergeb(en) 'mengampuni' +lich } & \text { vergeblich 'percuma,sia-sia' } \\ \text { ledig 'belum menikah' + lich } & \text { lediglich belaka' } \\ \text { froh'gembira'+lich } & \text { fröhlich 'gembira' } \\ \text { äußer'di luar' +lich } & \text { äußerlich'dilihat dari luar' } \\ & -i g\end{array}$

Die Morphologische die Adjektive mit dem Suffix -ig im Roman 'Eisprinzessin' von Carla Blumberg, die sie stammen aus Verben, Adjektiv, und Nomen, nämlich: 
Die Morphologische die Adjektive mit dem Suffix -isch im Roman 'Eisprinzessin' von Carla Blumberg bestehen aus Verben, Nomen, und Adjektive. Zum Beispiel:

$\operatorname{argwohn}(e n)$ 'mencurigakan' $+i s c h$

die Fantasie 'pantasi' + isch

die Sympathie 'simpati' $+i s c h$ argwöhnisch 'yang curiga'

fantastisch hayalan'

sympathisch'simpatik'

\section{-bar}

Die Morphologische die Adjektive mit dem Suffix -bar im Roman 'Eisprinzessin' von Carla Blumberg bestehen aus Adjektiv, Nomen, und Verben. Suffix -bar erklärt 'konnen'. Das sind:

die Sicht 'penglihatan' + bar

der Dank 'syukur'+bar

bemerk(en) ' mengetahui' +bar sichtbar 'dapat dilihat,tampak'

dankbar 'yang berterima kasih'

bemerkbar 'dapat diketahui'

\section{- los}

Die Morphologische die Adjektive mit dem Suffix -los im Roman 'Eisprinzessin' von Carla Blumberg stammen aus Nomen, Verben, und Adjektiv. Das sind:

das Verständnis ‘pengertian’+los

der Rat 'dewan' +los

nutz(en) 'berguna' +los

lust' kesenangan' + los

mühe 'mudah' +los verständnislos 'tidak pengertian' ratlos 'kehilangan akal' nutzlos 'tak berguna' lustlos 'bosan' mühelos 'gampang'

$$
\text { -voll }
$$

Die Morphologische die Adjektive mit dem Suffix -voll im Roman 'Eisprinzessin' von Carla Blumberg bestehen aus Nomen und Verben. Das sind: der Schwung 'ayunan'+voll schwungvoll 'bersemangat' 
die Pracht 'kemegahan'+voll

quäl(en) 'menyiksa' + voll prachtvoll 'sangat indah'

qualvoll ' yang menyiksa'

$-\ddot{o s}$

Die Morphologische die Adjektive von Suffix -ös im Roman ‘Eisprinzessin’ von Carla Blumberg besteht aus Nomen, zum Beispiel:

der Nerv 'urat saraf' $+\ddot{o s}$

nervös 'gugup'

\section{c. Interfiks}

Die Morphologische die Adjektive von Interfiks (Infiks) im Roman 'Eisprinzessin' von Carla Blumberg. Infiks im Roman gibt es $-\boldsymbol{s}$, und $-\boldsymbol{s t}$.

Das Element I Das Element II Die Vereinigung Die Bedeutung der Mann toll mannstoll 'gila laki-laki'

vorsicht halber vorsichtshalber 'supaya hati

hati'

\section{2) Kompotion}

Morphologischeprozess die Adjektive als Kompotion im Roman bestehen aus Adjektiv + adjektiv, Nomen + Adjektiv, und Verben + Adjektiv. Zum Beipiel:

$\begin{array}{ll}\text { fortschreitend } & \text { 'berlangsung terus' } \\ \text { lavendelblaue } & \text { 'lavendel kebiruan' } \\ \text { schlagartig } & \text { 'tiba-tiba' } \\ \text { wässrigblauen } & \text { 'air kebiru-biruan' } \\ \text { offensichtlich } & \text { 'nyata' } \\ \text { rückgängig } & \text { 'pembatalan' }\end{array}$

Diskussion 
Basierend auf den Ergebnisse des morphologische des Adjektiv wurde zusammengefasst, dass die Daten in dieser Untersuchung aus dem Roman, Internet und einigen Website genommen werden. In dieser Untersuchung wird Elke Hentschel Theorie benutzt. Dieser Untersuchung mit die Analyse Des adjektiv im Roman ist gut. Sodass die Dozenten oder die Studenten das als Lernmedia benutzen konnen.

\section{SCHLUSSFOLGERUNG}

Basierend auf dem Ergebnis der Analyse werden die folgenden Schlussfolgerungen gezogen:

1. Im Roman "Eisprinzessin" von Carla Blumberg gibt es viele Adjektive. Die Adjektive stammen aus Nomen, Adjektive, Adverbial, und Verben. Das Adjektiv hat die Morphologische. Die Morphologische des Adjektivs im Roman haben in zwei Teile geteilt, nämlich in Affix und Komposition. Affix besteht aus drei Teil. Das sind Präfix, Suffix und Interfiks. Präfix im Roman gibt es -un; Suffix im roman gibt es -lich, -isch, -ig, -bar, -sam, $\ddot{o} s$, -los, und -voll; und Interfiks gibt es $-s$ und $-s t$.

2. Nach der Untersuchung wird 253 die Morphologische Adjektive im Roman "Eisprinzessin" von Carla Blumberg gefunden. Es gibt 46 die Morphologische Adjektive mit dem Präfix (-un, -un + -lich, -un+-ig, un+-bar), 66 die Morphologische Adjektive mit dem Suffix -lich, 55 die Morphologische Adjektive mit dem Suffix -ig, 11 die Morphologische Adjektive mit dem Suffix -isch, 7 die Morphologische Adjektive mit dem Suffix -bar, 16 die Morphologische Adjektive mit dem Suffix -los, 9 die Morphologische Adjektive mit dem Suffix -voll, 3 die Morphologische Adjektive mit Suffix -sam und nur 1 die Morphologische Adjektive Suffix -ös. Im Roman gibt es Komposition. Die Morphologische die Adjektive als Komposition im Roman stammen aus Adjektiv + adjektiv, Nomen + Adjektiv, und Verben + Adjektiv.

\section{DAS LITERATURVERZEICHNIS}


Studia: Journal des Deutschsprogramms p-ISSN 2301-6108 e-ISSN 2654-9573

https://jurnal.unimed.ac.id/2012/index.php/studia/index

Bertelsmann. 2004. Grammatik Der Deutschen Sprache. Universitas Harvard. Germany.

Blumberg, Carla. 2014. Eisprinzessin: Roman. Münschen: Universo

Chaer, Abdul. 2001. Linguistik umum: Edisi Keempat. Jakarta: Rineka Cipta.

Heringer, Hans Jürgen. 2014. Deutsche Grammatik und Wortbildung in 125 fragen und Antworten.Stuttgart; Italier Reichrt

Kridalaksana, Harimurti. 2009. Kamus Linguistik: Edisi keempat. Jakarta : PT. Gramedia Utama Pustaka.

Miles und Huberman. 2014. Metode Penelitian Deskriptif. Jakarta : $\quad$ PT. Gramedia Utama Pustaka.

Siahaan,Jujur, S.Pd, M.Hum. 2013, Anlisis Morfologis Nomina Bahasa Jerman. Medan:

Unimed Press.

Sibarani, Robert. 2004. Antropologi Linguistik. Medan: Penerbit Poda

Internet:

Henschel, Elke. 2010. Deutsche Grammatik. De Gruyter. Germany, wird am 13 August 2017, um 20.22 gelesen.

Khoerudin, Ending. 2010. Adjektiva dalam Bahasa Jerman. Bandung. Jurusan Pendidikan Bahasa Jerman UPI. Wird am 30_August 2017, um 20.13 gelesen.

Okta, F. Filtas. 2012. Eprints . Uny.ac.id. Wird am 05 September 2017,um 16.30 gelesen. 